Video hilfreich für die diagnostische Einschätzung war. Zwölf bereits bekannte Patienten hatten Besonderheiten im Verlauf wie unklare anfallsartige Zustände dokumentiert. Aufgrund seiner Erfahrungen hält Mayer Homevideos für nützlich zur Klärung der Diagnose und von Medikamentenef- fekten. Auch die Notwendigkeit einer antiepileptischen Therapie oder der Einsatz von Notfallmedikamenten kann durch die Homevideos untermauert werden. Sie ermöglichen eine verbesserte Klassifikation epileptischer Anfälle sowie nicht epileptischer anfallsartiger Episoden und tragen dazu bei, die Häufigkeit eines EEG-Monitorings mittels Videoaufzeichnung $\mathrm{zu}$ senken.

Dr. Katharina Arnheim

8. Gemeinsame Jahrestagung der Deutschen und Österreichischen Gesellschaften für Epileptologie und der Schweizerischen Liga gegen Epilepsie, Interlaken/Schweiz, 8.-11.5.2013

\title{
Antikonvulsiva - nur jedes dritte Rezept bei Epilepsie
}

\author{
Das Verschreibungsmuster von Antikonvulsiva erlaubt Rückschlüsse auf die Prävalenz der Epilepsie in \\ Deutschland und liefert interessante Anhaltspunkte zu Charakteristik und Qualität der therapeutischen \\ Versorgung im ambulanten Setting.
}

$\mathrm{N}$ ur etwa ein Drittel der Antikonvulsiva in Deutschland werden an Epilepsie-Kranke verordnet, der Rest (64\%) an Patienten mit Schmerzsymptomen, bipolaren Störungen oder anderen psychiatrischen Erkrankungen. Besonders häufig in anderen Indikationen eingesetzt werden Pregabalin (96\%), Gabapentin (91\%), Carbamazepin (51\%) und Topiramat (41\%). Quelle für diese Zahlen sind die IMS ${ }^{\circledast}$ LRX-Datenbank und die Disease ${ }^{\circledR}$ Analyser-Datenbank der Bundesärztekammer [1].

Die Analyse der Daten identifizierte 634.566 Patienten, die 2009 Antikonvulsiva zur Behandlung ihrer Epilepsie eingenommen hatten. Daraus errechnet sich eine Periodenprävalenz von 0,9. Der Unterschied zur Punktprävalenz (0,5 in Europa laut einer aktuellen Erhebung) hängt nach Aussage von Professor Hajo Hamer, Erlangen, wahrscheinlich damit zusammen, dass sich mit dieser Metho- de nicht nur Patienten mit aktiver Krankheit, sondern auch solche ohne Anfallsaktivität erfassen lassen. Bestätigt hat sich der seit einigen Jahren beobachtete Trend, dass Menschen jenseits des 65. Lebensjahres häufiger an Epilepsie leiden (13 von 1.000) als Kinder und Jugendliche (5 von 1.000).

\section{Jeder zweite Epilepsie-Patient geht nur zum Hausarzt}

Überrascht hat Hamer die Tatsache, dass Antikonvulsivarezepte für EpilepsiePatienten häufiger vom Hausarzt ausgestellt werden als in der Nervenarztpraxis ( $56 \%$ vs. $47 \%$ ) und dass in $84 \%$ der Fälle nicht zwischen den Gesundheitssektoren gewechselt wird. Es gebe also relativ viele Epilepsie-Patienten, die nie von einem Spezialisten gesehen würden.

In Deutschland werden Epilepsie-Patienten noch immer deutlich häufiger mit Antikonvulsiva der ersten Generation

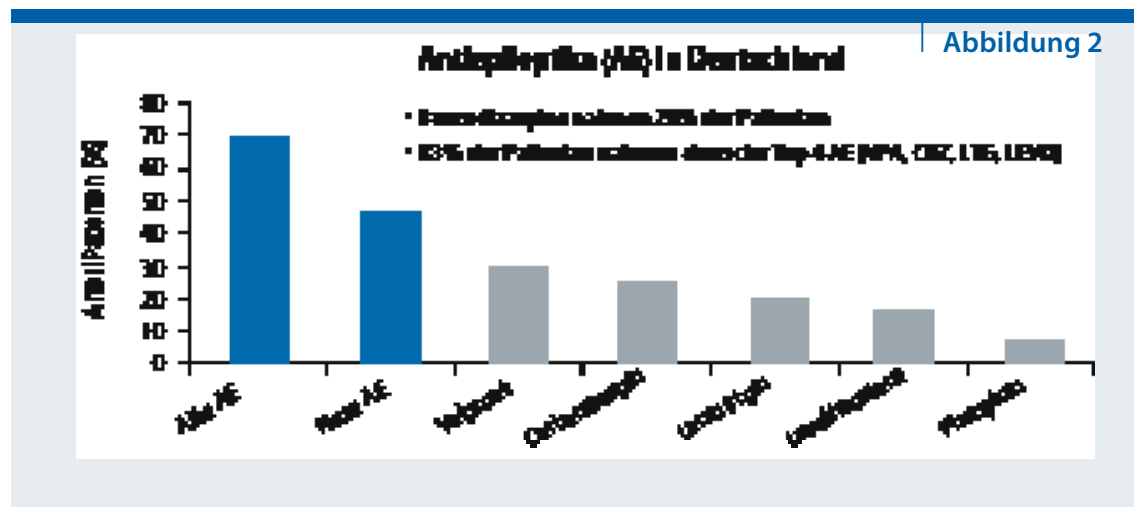

Verordnungshäufigkeit von Antikonvulsiva für Epilepsie-Patienten im Jahr 2009 in Deutschland [nach 1]

behandelt als mit modernen Substanzen. Spitzenreiter sind Valproat und Carbamazepin gefolgt von Lamotrigin und Levetiracetam (Abbildung 2). An fünfter Stelle steht Phenytoin. Dieses Antikonvulsivum spiele bei den Neurologen praktisch keine Rolle mehr, sei aber noch immer sehr beliebt bei den Hausärzten, zeigte Hamer die Zusammenhänge auf. Verordnet würde es vor allem Patienten im höheren Lebensalter, die wiederum die Majorität der Epilepsie-Kranken in der Hausarztpraxis stellten.

Als weiteres Phänomen stellte Hamer fest, dass 29\% der Epilepsie-Patienten zusätzlich zu Antikonvulsiva ein Benzodiazepin (vor allem Lorazepam und Diazepam) erhielten. Der Stellenwert dieser Substanzen als Notfallmedikation sei jedoch umstritten. Bei Einzelanfällen bringe der Einsatz nicht viel, ein Nutzen ist gegebenenfalls bei Anfallsserien oder Status epilepticus zu erwarten.

Für die an Epilepsie-Patienten verordneten Antikonvulsiva wurden von der GKV im Jahr 2009 rund 285 Millionen $€$ aufgewendet. Das sind im Median 158 $€$ pro Patient. Damit haben die Antikonvulsiva einen Anteil von nur etwa $1 \%$ an den Arzneimittelausgaben der GKV und von nur $0,1 \%$ an den Ausgaben für Gesundheit insgesamt, so Hamer.

Gabriele Blaeser-Kiel

1. Hamer HM et al. J Neurol 2012; 259: 2376-84

8. Gemeinsame Jahrestagung der Deutschen und Österreichischen Gesellschaften für Epileptologie und der Schweizerischen Liga gegen Epilepsie, Interlaken/Schweiz, 8.- 11.5.2013 